\title{
Stress relaxation of porcine tendon under simulated biological environment: experiment and modeling
}

\author{
SylWiA D. ŁAGAN, ANETA LIBER-KNEĆ* \\ Cracow University of Technology, Faculty of Mechanical Engineering, \\ Department of Applied Mechanics and Biomechanics, Kraków, Poland.
}

\begin{abstract}
Purpose: The aim of the study was to investigate the viscoelastic response in the low and high physiological strain with the use of experimental and modeling approach. Methods: Viscoelastic response in the low, transition and high physiologic strain (3, 6 and 9\%) with consideration of simulated biological environment $\left(0.9 \%\right.$ saline solution, $\left.37{ }^{\circ} \mathrm{C}\right)$ was measured in relaxation tests. Preconditioning of tendons was considered in the testing protocol and the applied range of load was obtained from tensile testing. The quasi-linear viscoelasticity theory was used to fit experimental data to obtain constants (moduli and times of relaxation), which can be used for description of the viscoelastic behavior of tendons. The exponential non-linear elastic representation of the stress response in ramp strain was also estimated. Results: Differences between stress relaxation process can be seen between tendons stretched to the physiological strain range (3\%) and exceeding this range $(6$ and $9 \%)$. The strains of $6 \%$ and $9 \%$ showed a similar stress relaxation trend displaying relatively rapid relaxation for the first 70 seconds, whereas the lowest strain of $3 \%$ displayed relatively slow relaxation. Conclusions: Results of the model fitting showed that the quasi-linear viscoelastic model gives the best fit in the range of low physiological strain level.
\end{abstract}

Key words: tendon, stress relaxation, quasi-linear viscoelasticity, simulated biological environment

\section{Introduction}

The mechanical properties of tendons were widely investigated in different species and in different anatomical locations [6], [16]. Mechanical behavior of tendons is described as inhomogeneous, anisotropic, nonlinear and viscoelastic [3], [20]. Tendons are adapted to carry repeated loads and are known to adapt to mechanical loading. Also, their healing and disease processes are highly sensitive to mechanical load. The most common mechanical tests for tendons include tensile tests, creep and relaxation tests. Tensile tests are used to examine general mechanical properties of tendons including: ultimate tensile strength (UTS), Young's modulus and the stress-strain curve. A typical stress-strain curve of tendon includes four regions
[14]. First is the toe region, in which the tendon is strained up to $2 \%$. Following the toe region, there is a linear region which ranges between $2 \%$ and $6 \%$ strain. The physiological tensile range of tendon during daily activities lies in this region but it must be remembered that molecular damage is still possible and depends on loading. When strain is larger than 6\% (i.e., third region), partial damage of tendon fibers occurs. With further increase of strain above $10 \%$, macroscopic tearing of tendon fibers leads to tendon rapture. Values reported in literature show a great deal of variation in human tendon properties, e.g., different types of tendons differ greatly in their mechanical properties. For example, Young's modulus of the human patellar tendon is $660 \pm 266 \mathrm{MPa}$ (mean $\pm \mathrm{SD}$ ) [14], whereas for the Achilles tendon it is $816 \pm 218 \mathrm{MPa}$ [25]. Ultimate tendon stress values are reported in the

\footnotetext{
* Corresponding author: Aneta Liber-Kneć, Cracow University of Technology, Department of Applied Mechanics and Biomechanics, al. Jana Pawła II 37, 31-864, Kraków, Poland. Phone: +48 692586 152, e-mail: aliber@pk.edu.pl

Received: October 15th, 2020

Accepted for publication: December 18th, 2020
} 
range between 50 to $100 \mathrm{MPa}$ and ultimate tendon strain values are between $4 \%$ and $10 \%$ [17]. Failure forces of up to $7300 \mathrm{~N}$ have been documented [25].

Within body, tendons are stretched in a wide range of strain rates and their viscoelasticity affects the ability to store and dissipate energy, and adapt to loading conditions over time. Thus, investigating the viscoelastic properties of tendons is very important to assess their mechanical integrity and to prevent their injuries. Viscoelasticity makes tendons more deformable at low strain rates, therefore tendons tend to absorb more mechanical energy, but are less effective in carrying mechanical loads. On the other hand, at high strain rates, tendons are less deformable, become stiffer and more effective in transmitting large muscular loads to bones. Stress relaxation is attributed to the rearrangement of the microstructure allowing for a redistribution of stress [14], [21]. Constitutive modeling can be a complementary to experimental studies in the process of evaluating tendon mechanical properties under normal function, the mechanism of damage and healing, as well as capturing mechanobiological adaptation in tendons [6].

Mathematical models of tendon mechanics represent two general approaches: phenomenological and microstructural, and are important to estimate forces occurring during locomotion activities. The phenomenological approach uses mathematical formulations that can closely match tendon mechanical responses on the tissue scale. The microstructural approach describes complete tendon mechanical behavior by combining or generalizing the mechanical behavior of different components in tendon. Wide review of literature included in [11] outlines mathematical and computational models used to understand tendon mechanics at different scales of the hierarchical organization. Tendon tissue, due to its complex structure, exhibits the properties of orthotropic heterogeneous material, but for the purposes of mathematical descriptions, simplification such assumptions of their anisotropy or isotropy are often made. Many models of phenomenological approach were developed based on the continuum theory to emphasize tendons hyperelastic, viscoelastic and poroelastic properties [9], [15], [17], [21]. In order to describe the biomechanical behavior of tendons at the microscopic level, with consideration of collagen fibrils structure, the mathematical formulation used to represent tendon behavior was based on a continuum assumption on the tissue scale [3], [4], [20]. In addition, several different modeling schemes were developed to evaluate mechanical properties of individual tendon constituents, or their interactions, on the molecular level [18].

One of the most commonly employed model of tendons is the quasi-linear viscoelastic (QLV) theory developed by Fung [12]. The QLV model is attractive due to ease of implementation. Quasi-static tensile tests and stress relaxation tests at a single strain level are sufficient to compute its parameters. The QLV model has been used to fit experimental data in numerous studies of soft tissues [6], [14], however, recent investigations have questioned the ability of this model to accurately predict stresses or strains under conditions other than those used to fit the model [5], [8]. As alternatives to the QLV theory, nonlinear superposition [19] and Schapery nonlinear equations, and other single or multiple integral models [8] have been proposed. The analysis of the modeling results presented in literature, shows that the scope of application of described material models should be carefully determined, due to the possibility of obtaining divergent results depending on the experimental protocol and data range used. Especially, when it is difficult to clearly define the boundaries of small and large deformation ranges for the examined tissues and the elastic limit of the biological material.

The goal of this study was to investigate the viscoelastic response in the low and high physiological strain with the use of experimental and modeling approach. To determine nonlinear viscoelastic properties of specimens being tested, relaxation tests were conducted at three strain levels. An important issue in this investigation was the incorporation of simulation of biological environment and preconditioning process of samples into the experimental protocol. A lot of experiments reported in literature [7] are conducted without simulation of body environment conditions (room temperature and humidity) despite the fact that specimens hydration influences on stress relaxation of tissues. The experimental data from stress-relaxation tests were used in fitting the QLV theory to obtain constants which can be used for description of the viscoelastic behavior of tendons. Considering the negative and positive opinions regarding the QLV, our aim was to investigate, whether the ability of this model to match experimental data depends on the level of strain used in direct stress relaxation tests, each conducted at a single axial stretch.

\section{Materials and methods}

\subsection{Sample preparation}

The object of research were tendons (tendo calcaneus communis) extracted from legs of domestic pigs. 
The biological material was isolated as a food waste from a local abattoir. Until the time of examination (no longer than 24 hours) samples were stored in $0.9 \%$ saline solution at $4{ }^{\circ} \mathrm{C}$. Dimensions of the crosssection were measured along the major axis (transversal diameter) and the small axis (conjugate diameter) at three equidistant locations along the length of the tendon with the use of a digital caliper. Tendons cross-sectional area was calculated assuming an elliptical cross section with the use of the averages of diameters. The assumption of the elliptical cross-section resulted from rounded edges of specimens and more precise measurements of diameters compared to the measurements of specimen sides for a rectangular cross-section. The average cross-section area of tendons was $40.48 \pm 8.71 \mathrm{~mm}^{2}$.

\subsection{Tensile and stress relaxation tests}

All tests were conducted using the MTS Insight 50 tensile machine in combination with TestWorks4 software for data collection. Samples were mounted in self-tightening scissors grips to ensure suitable and secure clamping. To simulate the body environment, an environmental chamber with 10.0 liters of $0.9 \%$ saline solution heated to body temperature $\left(37 \pm 1{ }^{\circ} \mathrm{C}\right)$ was used (Fig. 1). Preliminary uniaxial tensile tests were conducted before the stress relaxation tests, to characterize the mechanical parameters of tendon

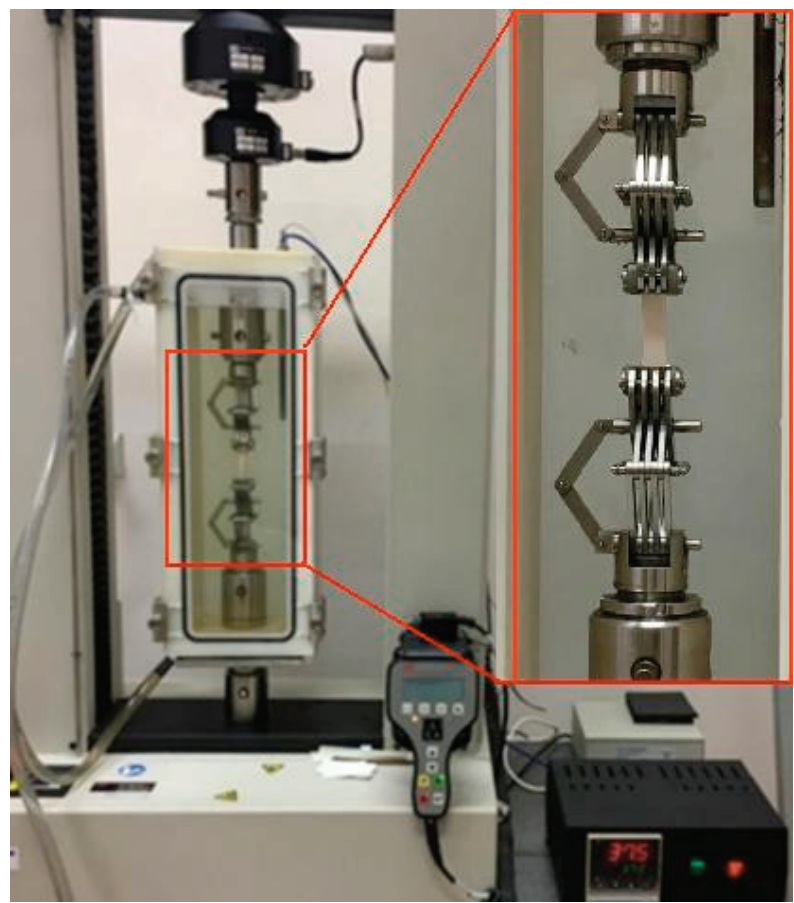

Fig. 1. The MTS tensile machine with the environmental chamber heated to $37{ }^{\circ} \mathrm{C}$ used in tests tissue (ultimate tensile strength, Young's modulus) and to identify the best load and loading rate in relaxation tests. The uniaxial tensile tests were conducted at a constant rate of $5 \mathrm{~mm} / \mathrm{min}$, for ten samples. The length of the measurement base $\left(l_{0}\right)$ was $50 \mathrm{~mm}$. Registered force $(F)$ - elongation $(\Delta l)$ curves were recalculated into stress $(\sigma)$ - strain $(\varepsilon)$ curves.

According to the literature [22], [23], preconditioning significantly influences tendon mechanical properties and sets up the same initial properties for all specimens. Before each stress relaxation test, tendons were preconditioned in the load controlled experiment. The applied range of load was obtained from tensile tests. The upper limit of load was taken to ensure that the strain remained within the linear region. The maximum load of each load-unload cycle was fixed at $5 \mathrm{~N}$. The length $\left(l_{0}\right)$ of the measurement base was $30 \mathrm{~mm}$. Loading and unloading were repeated until the stress-strain loop of the sample reached a steady state, understood as the same shape of the loops and value of dissipation energy calculated as the area of the loop. This was achieved after 3 to 5 cycles of loading-unloading for each sample. Tendons were allowed to recover for 10 minutes before relaxation testing started. Next, a $2 \mathrm{~N}$ preload was applied to remove any slack in the tendon and to get close to its natural state of pretension. Direct stress relaxation tests, each at a single axial stretch of 3,6 and 9\% were conducted. Samples were subjected to a ramp of 1.0, 2.0 and $3.0 \mathrm{~mm}$ grip-to-grip displacement at a strain rate of $0.5 \% / \mathrm{s}$, followed by a $300 \mathrm{~s}$ hold. The levels of strain were selected based on stress-strain curve of tendon as strain in the range of low physiological strain $(3 \%)$, high physiological strain $(6 \%)$, but still being low enough to avoid damage or failure, and strain resulting in the tendon rupturing (9\%) [12], [24]. An analysis of results was conducted for 12 specimens (4 for each level of strain) and the average stress relaxation curve was obtained for each strain level. The stress relaxation curves were shown as a dependence of true stress and real time. For the need of model fitting, the true stress was recalculated into normalized stress according to Eq. (3). Percent stress loss (PSL) was calculated over the $300 \mathrm{~s}$ relaxation test as:

$$
P S L=\frac{\sigma_{0.1 \mathrm{~s}}-\sigma_{300 \mathrm{~s}}}{\sigma_{0.1 \mathrm{~s}}}
$$

where $\sigma_{0.1 \mathrm{~s}}$ and $\sigma_{300 \mathrm{~s}}$ are the stresses at $0.1 \mathrm{~s}$ and $300 \mathrm{~s}$, respectively. Isochronal curves were conducted for $0.1,1,10,100,200$ and $300 \mathrm{~s}$ to compare the values of relaxation stress on different stages of relaxation process of tendons stretched to three levels of strain. 


\subsection{Quasi-linear viscoelastic characteristic}

The QLV theory models the viscoelastic response of a material based on the instantaneous elastic response resulting from a ramp strain and the reduced relaxation function [12]:

$$
\sigma(t)=G(t) \cdot \sigma^{\mathrm{e}}(\varepsilon),
$$

where, $\sigma(t)$ is the stress at any time, $\sigma^{\mathrm{e}}(\varepsilon)$ is the stress corresponding to an instantaneous strain and $G(t)$ is the reduced relaxation function. The reduced relaxation function $G(t)$ represents the stress of the material divided by the stress after the initial ramp strain (3):

$$
\begin{gathered}
G(t)=\frac{\sigma(t)}{\sigma_{\max }}, \\
G(0)=1 .
\end{gathered}
$$

The stress responses to a small strain increment, applied at time $\tau$, can be assumed using the Boltzman superposition principle, so that the stress for $t \geq 0$ can be written as:

$$
\sigma(t)=\int_{0}^{t} G(t-\tau) \frac{\partial \sigma^{\mathrm{e}}(t)}{\partial \varepsilon} \frac{\partial \varepsilon}{\partial \tau},
$$

where, $\frac{\partial \sigma^{\mathrm{e}}(t)}{\partial \varepsilon}$ is the temporary elastic response, and $\frac{\partial \varepsilon}{\partial \tau}$ is the strain history of the sample. The reduced relaxation function is:

$$
G(t)=A_{0}+A_{1} e^{-t / \tau_{1}}+A_{2} e^{-t / \tau_{2}},
$$

where, $A_{0}, A_{1}$ and $A_{2}$, are model constants which can be determined based on experimental data curve; $\tau_{1}$ and $\tau_{2}$ are time constants, associated with relaxation process occurring at different time scales. An exponential non-linear elastic representation of the stress response is given as:

$$
\sigma^{\mathrm{e}}(\varepsilon)=A\left(\mathrm{e}^{B \varepsilon}-1\right),
$$

where, $A$ is a linear parameter which has the same dimension as stress, and $B$ is non-dimensional factor describing the nonlinearity of the elastic response. $A$ and $B$ are constants to be fitted with experimental data. Reduced relaxation function (Eq. (6)) was first fitted to the stress-relaxation data measured at 3, 6 and 9\% strain to determine the material constants with OriginPro software. The reduced chi-squared $\chi_{\text {red }}^{2}$ was calculated according to:

$$
\chi_{\text {red }}^{2}=\frac{\chi^{2}}{d},
$$

where, $\chi_{\text {red }}^{2}$ is chi-squared and $\mathrm{d}$ is the number of degrees of freedom.

\section{Results}

The mechanical behavior of tendon under tensile tests showed typical for tendon tissue shape (Fig. 2) [14]. The mechanical behavior of tendon was identified both in uniaxial tensile tests and relaxation tests, in order to describe material parameters. The obtained value of ultimate tensile strength was $21.5 \pm 7.5 \mathrm{MPa}$, Young's modulus was $339.9 \pm 94.4 \mathrm{MPa}$ and strain at ultimate strength was $12 \%$. The borders of regions I, II, III, and IV identified for the average stress-strain curve were well matched with the typical stress-strain curve reported by Johanson et al. [14] for human patellar tendon.

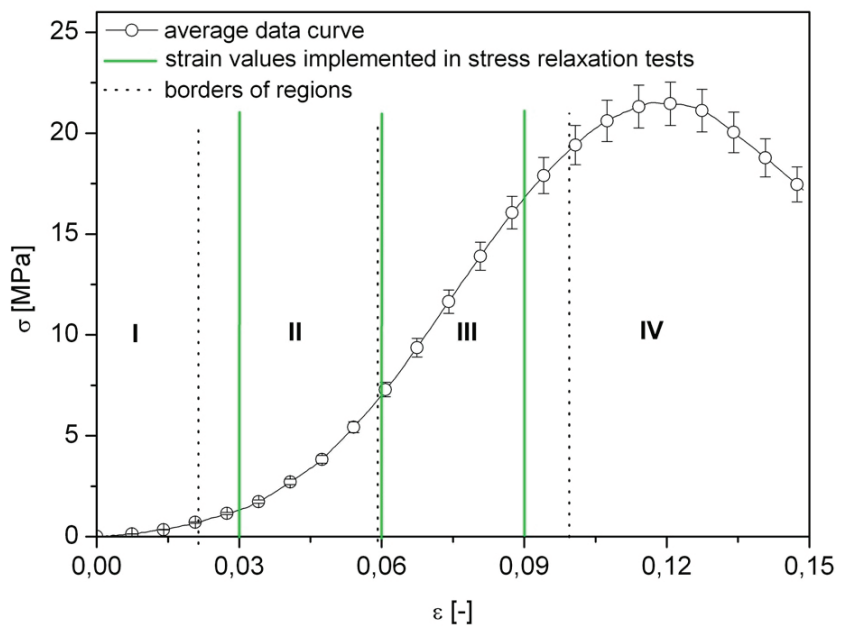

Fig. 2. The average stress-strain curve of tendons with distinguished four regions and marked the strain values used in stress relaxation tests

Stress relaxation data were obtained by subjecting tendons to constant displacement from 1.0 to $3.0 \mathrm{~mm}$. Results of stress relaxation experiments performed at various strain levels are shown in Fig. 3a. The stress relaxation data normalized by the initial stress value (Eq. (3)) are presented in Fig. 3b. Four characteristic phases of tendons stress relaxation can be distinguished: the fast relaxation region, the transition region, the slow relaxation and the equilibrium state region. Differences between stress relaxation process can be seen between tendons stretched to the physiological strain range and exceeding this range. The strains of $6 \%$ 
and $9 \%$ showed a similar stress relaxation trend displaying relatively rapid relaxation for the first $70 \mathrm{sec}-$ onds, whereas the lowest strain of 3\% displayed relatively slow relaxation. The decaying rate was reduced after this time and reached a similar level for all strains. For samples strained to $6 \%$ and $9 \%$, stress decayed to a normalized stress of approximately 0.2 , whereas for samples strained to $3 \%$ it decayed to a normalized stress of approximately 0.3 . In the fast relaxation region, difference between stress values for samples strained to 3,6 and 9\% was $0.2 \mathrm{MPa}$ and decreased in time of relaxation. Percent stress loss was

Table 1. Constants describing the instantaneous elastic response obtained by curve-fitting

\begin{tabular}{|c|c|c|c|}
\hline $\begin{array}{c}\text { Strain level } \\
{[\%]}\end{array}$ & $\begin{array}{c}A \\
{[\mathrm{MPa}]}\end{array}$ & $B$ & $R^{2}$ \\
\hline 3 & $0.1149 \pm 0.0034$ & $51.2788 \pm 0.8058$ & 0.9986 \\
\hline 6 & $8.5621 \pm 4.1951$ & $1.5178 \pm 0.7157$ & 0.9907 \\
\hline 9 & $10.7570 \pm 6.6443$ & $1.2908 \pm 0.7593$ & 0.9625 \\
\hline
\end{tabular}

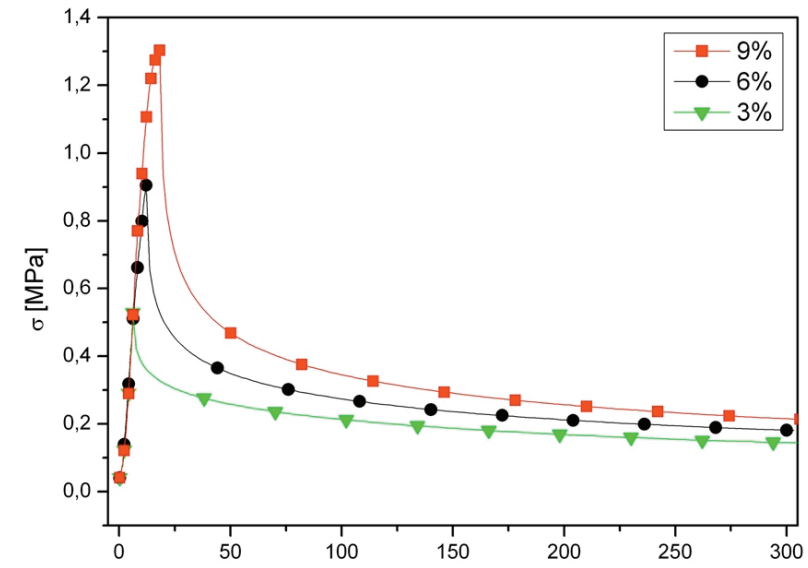

(a)

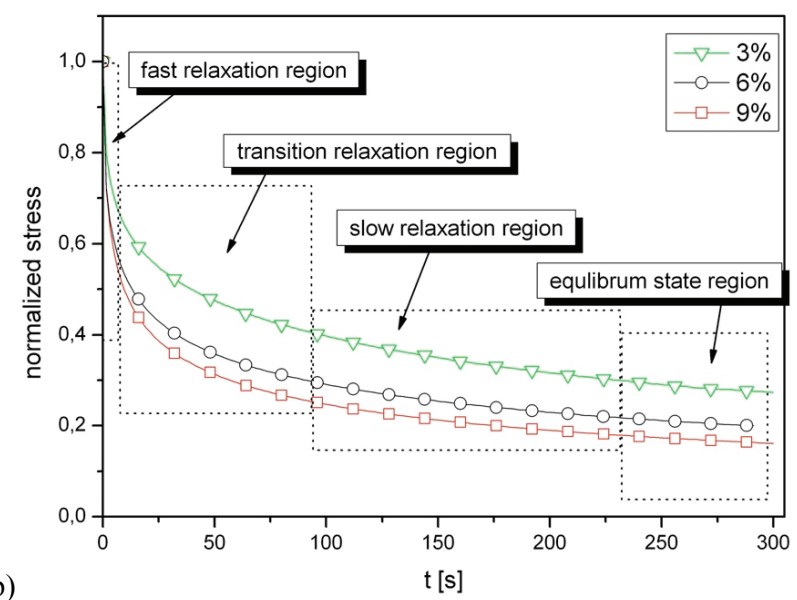

Fig. 3. Representative ramp and relaxation curves for each strain level (a), representative curves ramp and relaxation curves for each strain level (relaxation curves recalculated to normalized stress) (b)

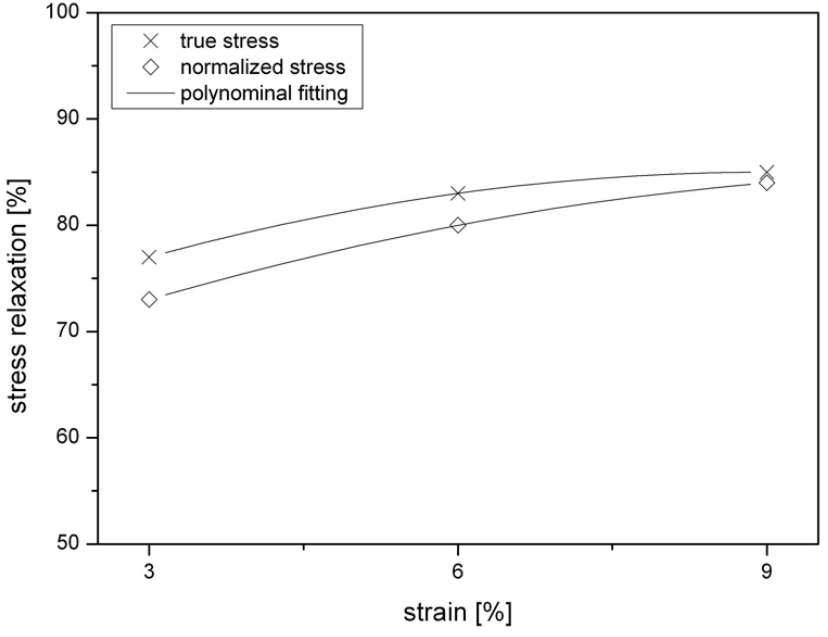

Fig. 4. Percent stress loss at 300 seconds

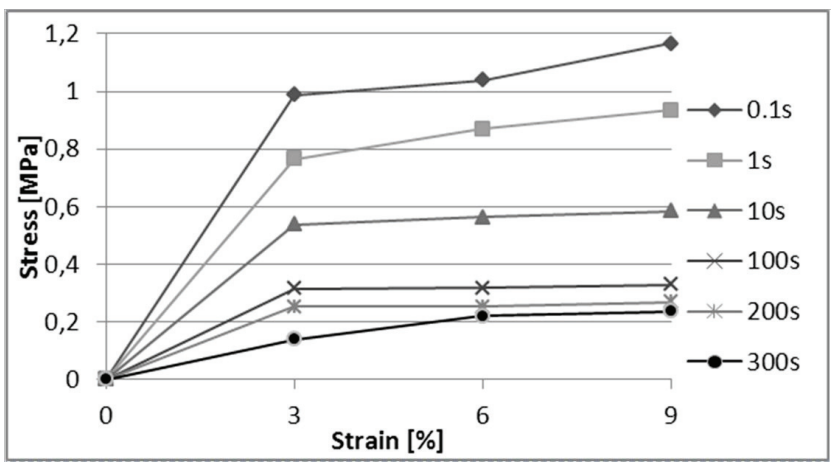

Fig. 5. Representative isochronal results obtained from relaxation tests

highest for strain level $9 \%$ and was $86.7 \%$, for strain levels 3 and $6 \%$, it was 79.8 and $80.0 \%$, respectively (Fig. 4). A positive quadratic correlation $\left(R^{2}=0.99\right)$ was observed between the value of percent stress loss observed at 300 seconds (from peak of observed stress) and individual levels of strain. Isochronal curves were generated from a set of points at the same times $(0.1$, 1, 10, 100, 200 and $300 \mathrm{~s}$ ) and applied strain levels within a set of relaxation curves (Fig. 5). Isochronal curves present the range of stress for which relaxation tests were performed, displaying the strain-stiffening behavior of tendon. Comparison of stress values in different time periods showed no significant effect of strain level between higher strain levels (6 and 9\%) but between strain level of 3 and $9 \%$, the difference of stress value was more visible, it was in the range of 0.1 to $0.2 \mathrm{MPa}$, depending on time. After $300 \mathrm{~s}$ of stress relaxation, the stress value was $0.13,0.22$ and 0.23 for strain levels 3, 6 and $9 \%$, respectively.

A typical curve fitted to the stress relaxation of the experimental data is shown in Fig. 6, where actual stress (Fig. 6a) and normalized stress (Fig. 6b) are plotted against time. The best fit between experi- 

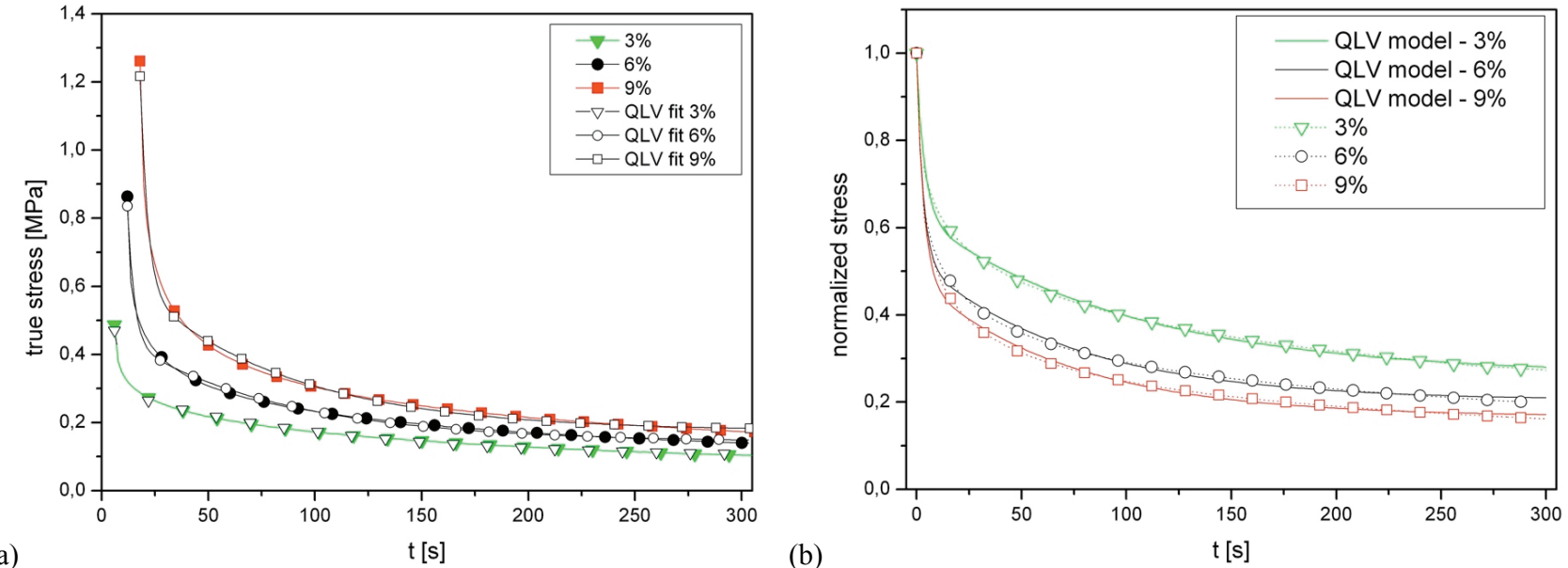

Fig. 6. Fitting QLV model curves for pig tendon: a) time vs true stress, b) time vs normalized stress

Table 2. Constants describing the reduced relaxation function obtained by curve-fitting (normalized stress)

\begin{tabular}{|c|c|c|c|c|c|c|c|}
\hline$\%$ & $A_{0}$ & $A_{1}$ & $A_{2}$ & $\tau_{1}[\mathrm{~s}]$ & $\tau_{2}[\mathrm{~s}]$ & $\chi^{2}$ & $R^{2}$ \\
\hline 3 & $0.261 \pm 0.002$ & $0.342 \pm 0.072$ & $0.364 \pm 0.003$ & $3.882 \pm 0.164$ & $101.982 \pm 2.310$ & 0.0001 & 0.9956 \\
\hline 6 & $0.204 \pm 0.002$ & $0.445 \pm 0.092$ & $0.321 \pm 0.037$ & $3.085 \pm 0.131$ & $75.255 \pm 2.021$ & 0.0001 & 0.9928 \\
\hline 9 & $0.166 \pm 0.002$ & $0.484 \pm 0.009$ & $0.315 \pm 0.004$ & $3.442 \pm 0.132$ & $72.179 \pm 1.910$ & 0.0001 & 0.9932 \\
\hline
\end{tabular}

Table 3. Constants describing the reduced relaxation function obtained by curve-fitting (true stress)

\begin{tabular}{|c|c|c|c|c|c|c|c|}
\hline$\%$ & $A_{0}$ & $A_{1}$ & $A_{2}$ & $\tau_{1}[\mathrm{~s}]$ & $\tau_{2}[\mathrm{~s}]$ & $\chi^{2}$ & $R^{2}$ \\
\hline 3 & $0.097 \pm 0.001$ & $0.859 \pm 0.062$ & $0.204 \pm 0.001$ & $3.893 \pm 0.163$ & $102.074 \pm 2.315$ & 0.0002 & 0.9956 \\
\hline 6 & $0.143 \pm 0.002$ & $20.521 \pm 3.487$ & $0.341 \pm 0.005$ & $3.075 \pm 0.128$ & $75.175 \pm 2.015$ & 0.0007 & 0.9928 \\
\hline 9 & $0.175 \pm 0.002$ & $118.528 \pm 23.750$ & $0.527 \pm 0.009$ & $3.449 \pm 0.128$ & $72.248 \pm 1.912$ & 0.0002 & 0.9932 \\
\hline
\end{tabular}

mental and model curve can be seen for the lowest strain level (3\%). These data were fit with an overall $R^{2}$ value greater than 0.99 . Good fit between experimental and model curves was also observed for the level of strain $6 \%\left(R^{2}=0.99\right)$. Similarly, for tendons stretched to the physiological strain range ( 3 and $6 \%$ ), the instantaneous assumption approach enabled to obtain separate fits of these data with good quality $\left(R^{2}=0.99\right)$ (Table 1$)$. The parameters $A$ and $B$ in nonlinear material model of stress-strain behavior showed strong dependence of strain level. Larger change (in the value) of the $A$ parameter (increase by $98 \%$ ) was observed between 3 and $6 \%$ strain level rather than between the 6 and $9 \%$ strain level (increase by 20\%). The change of the $B$ parameter value showed a reverse trend, a decrease by $97 \%$ between the 3 and $6 \%$ strain level, and $13 \%$ between the 6 and 9\% strain level (Table 1). The constants describing the reduced relaxation function, were compared in Tables 2 and 3. The values of constant $\tau_{1}$ were similar for each strain level which indicates that the short-term relaxation behavior is not affected by the strain level. The differences between constants $\tau_{2}$ for specimens stretched to physiological strain $(3 \%)$ and higher strains (6 and 9\%) indicate that stress relaxation reaches a plateau earlier for high levels of strain. The dimensionless constants $A_{0}, A_{1}$ and $A_{2}$, i.e., the magnitude of the viscous response, were in a similar range for all levels of strain (Table 2). The stiffness as $A_{0}, A_{1}$ and $A_{2}$ were not proportional to each other (Fig. 7 , Tables 4 and 5).

Table 4. Modulus $A_{i}$ for QLV material model (normalized stress) representing the strain-dependent behavior

\begin{tabular}{|c|c|c|c|}
\hline Modulus $A_{i}$ & $a_{0}$ & $a_{1}$ & $a_{2}$ \\
\hline$A_{0}$ & 0.337 & -0.028 & 0.001 \\
\hline$A_{1}$ & 0.175 & 0.066 & -0.003 \\
\hline$A_{2}$ & 0.444 & -0.033 & 0.002 \\
\hline
\end{tabular}

Table 5. Modulus $A_{i}$ for QLV material model (true stress) representing the strain-dependent behavior

\begin{tabular}{|c|c|c|c|}
\hline Modulus $A_{i}$ & $a_{0}$ & $a_{1}$ & $a_{2}$ \\
\hline$A_{0}$ & 0.03729 & 0.02212 & $-7.59444 \mathrm{E}-4$ \\
\hline$A_{1}$ & 59.54166 & -32.61811 & 4.35246 \\
\hline$A_{2}$ & 0.11595 & 0.02137 & 0.00271 \\
\hline
\end{tabular}


The strain-dependent behavior of modulus was approximated by a five-term Prony series, where each strain-dependent Prony weight was described with a second order polynomial formulation:

$$
A_{i}(\varepsilon)=a_{0}+a_{1} \varepsilon+a_{2} \varepsilon^{2} .
$$

All moduli (Fig. 7) were fitted as a function of strain level from relaxation tests. The strain-dependent moduli components were described well (Tables 4 and 5) with $R^{2}=0.976$. The strain-dependent moduli $A_{1}$ showed non-linear characteristics, however, in the case of $A_{0}$ and $A_{2}$, discussion regarding non-linearity remains open. A linear interpolation conducted for moduli $A_{0}, A_{1}$ and $A_{2}$ showed that its implementation gives similar results as quasi-linear fitting of moduli $A_{0}$ and $A_{2}$ with coefficient of determination $R^{2}=0.996$. Only modulus $A_{1}$ showed clear non-linear characteristic with $R^{2}$ for linear interpolation equal to 0.871 . It showed the possibility of using a quasi-linear model in predicting stressrelaxation behavior of tendon tissue.

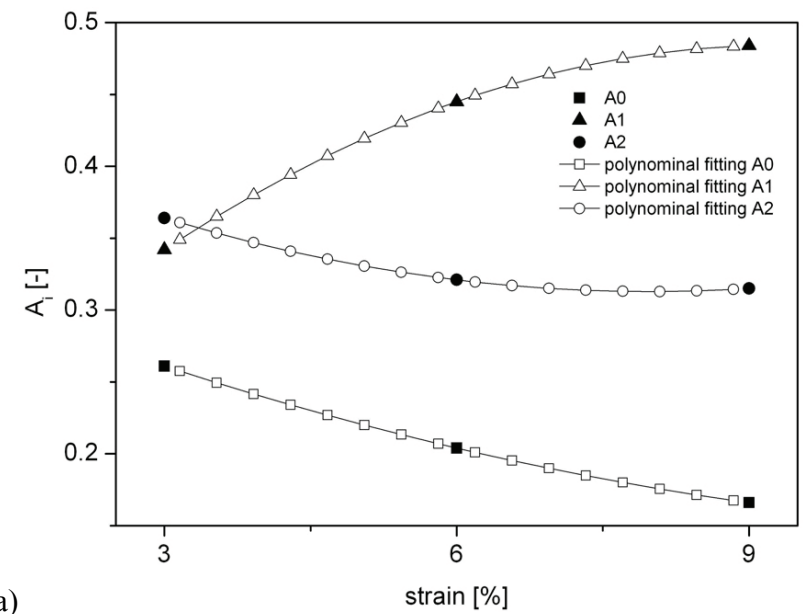

(a)

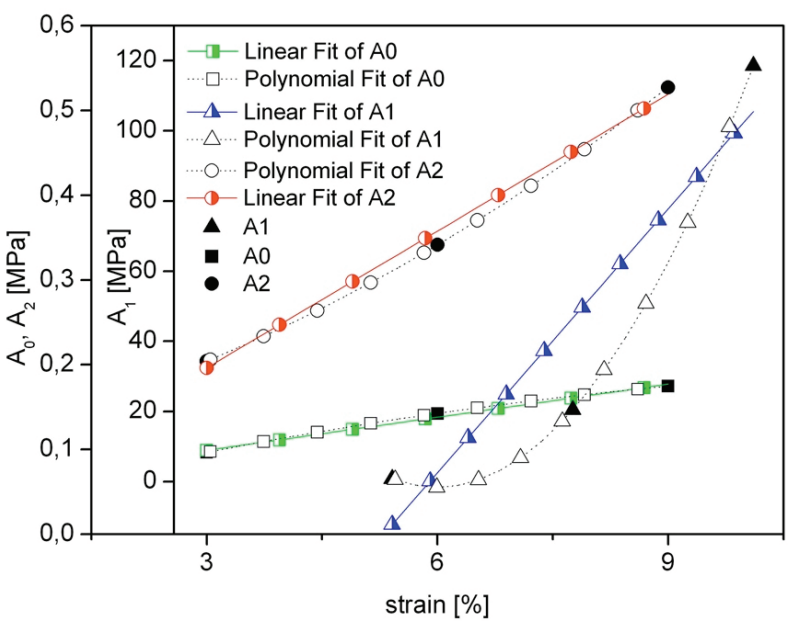

Fig. 7. The strain-dependent behavior of modulus described with a second order polynomial formulation for: (a) normalized stress, (b) true stress
Analysis of the distribution of the QLV model fit error to stress curves (Fig. 8) revealed four local minima which correspond to phases of relaxation process. The first value of time (approx. $25 \mathrm{~s}$ ) did not cover the beginning of the transition relaxation region, but other obtained values of time coincide with the boundaries of the relaxation stages highlighted in Fig. 3b. Local minima of error curves correspond to values of time for which the lowest error of fitting was achieved. Mismatch of the first local minima with the beginning of the fast relaxation region resulted from the difficulty of clear determination of particular regions of relaxation.

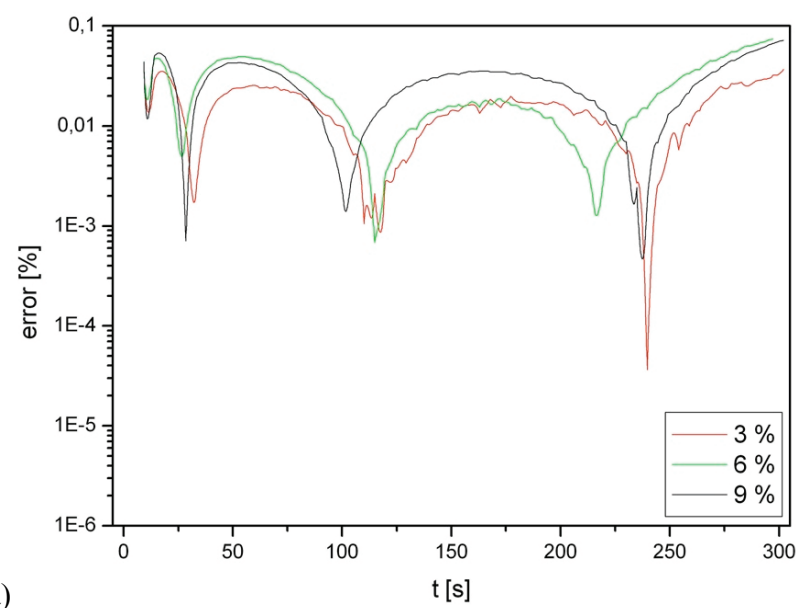

(a)

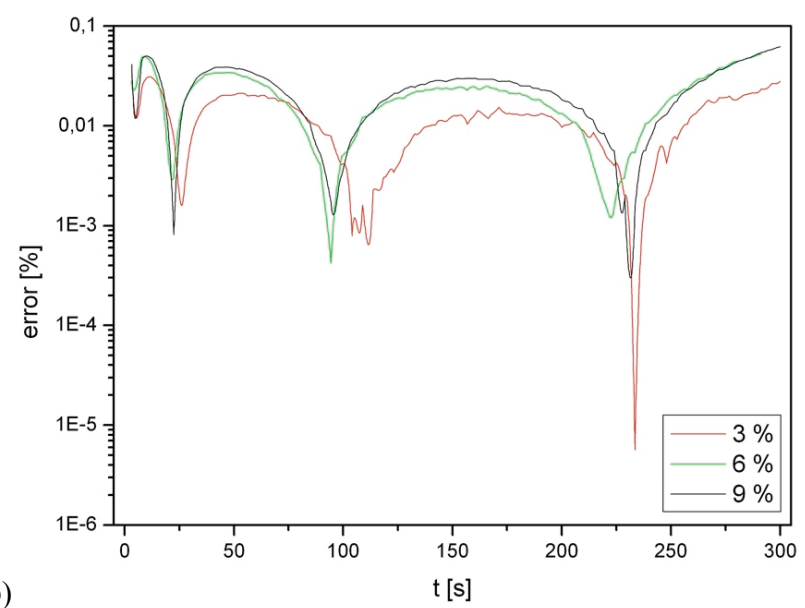

Fig. 8. The distribution of QLV models fitting errors for pig tendon for: (a) true stress, (b) normalized stress

\section{Discussion}

In this paper, the stress relaxation process and the ability to predict stress relaxation behavior in the low and high physiological strain was estimated. Due to the legal regulations and ethical reasons obtaining hu- 
man tissue for experimental research is difficult. Therefore, the material of animal origin was used as a substitute for qualitative and quantitative studies and modeling in this research. The QLV constitutive model evaluated in this study was chosen because it is a single integral model, and, therefore, is relatively easy to calculate, especially in step loading inputs used in stress relaxation testing. As the strain level increased, the ability of the QLV model to accurately predict stress relaxation decreased. Direct stress relaxation tests, each at a single axial stretch, were conducted to provide more accurate information about the effect of strain on stress relaxation. Additionally, experiments were conducted in a simulated biological environment, also including preconditioning to the experimental protocol in an attempt to provide a more consistent strain history and reference state. Different approaches utilized in others studies makes discussion with the available literature difficult. According to Maganaris, tendons of equal lengths but different cross-sectional areas show different force-deformation properties, and thicker tendons are stiffer. Similarly, different forcedeformation curves are obtained from tendons of equal cross-sectional areas, but different initial lengths, in which case shorter tendons are stiffer [16], [17]. The values of ultimate tensile strength and Young's modulus reported in [25] for Achilles tendon were $71 \pm 17$ and $816 \pm 218 \mathrm{MPa}$, additionally failure strain was 12.8 $\pm 1.7 \%$. Earlier studies [16] conducted on pig and sheep tendons, confirmed the hyperelastic behavior of tissues and allowed to determine the values of ultimate tensile strength and Young's modulus for pigs equal to $8.4 \pm 3.4 \mathrm{MPa}$ and $77 \pm 22 \mathrm{MPa}$, respectively (with average cross-section equal to $20 \mathrm{~mm}^{2}$ ) and for sheep equal to $17.5 \pm 7.4 \mathrm{MPa}$ and $156 \pm 57 \mathrm{MPa}$, respectively (with average cross-section equal to $29 \mathrm{~mm}^{2}$ ).

Strains used in this study were within the second region (linear) and the third region of the stress-strain curve. It is thought that tendon usually elongates 3-4\% during physiological loading, however tendon strains up to $10 \%$ for the Achilles were reported [9]. Thus, it was assumed that a grip-to-grip strain of 3, 6 and $9 \%$ would allow stress relaxation of the tissue. The isochronal curves which display values of stress during stress relaxation tests, indicated that tendon is in the "toe" region of the stress-strain curve. The stress induced at $9 \%$ strain was much lower than the ultimate stress of the tendon, which was $21.5 \pm 7.5 \mathrm{MPa}$. The stress-relaxation test showed increase of rate of relaxation for high strain levels compared to lower strain level. This behavior is similar to results found for porcine digital flexor tendon [9], but for the human MCL [2] or the rat Achilles tendon was opposite [15].
The variations in results could be caused by the origins of the tendons used in the studies as tendons of various anatomical sites have different functions and thus different biomechanical behavior.

The best prediction of the stress relaxation process was obtained for the lowest level of strain (3\%). Similar observations about the QLV prediction below $3 \%$ strain were reported by DeFrate et al. [6] for patellar tendon. Duenwald et al. [8] reported that stress relaxation is modeled well by the QLV for porcine digital flexor tendon for strains in the range of 1 to $6 \%$ but only at one level of strain. Application of multiple strain levels in relaxation experiments excludes the use of the QLV model. Constants describing the instantaneous elastic response and the reduced relaxation function obtained by curve-fitting differed in dependence on the level of strain. The range of values reported in literature for tendons for the constants $A$, $B, \tau_{1}$ and $\tau_{2}$ of the QLV theory is wide. The $A$ values were reported between 0.5 and $281 \mathrm{MPa}$, the $B$ value was from 2.11 to 56.3 , the range of the $\tau_{1}$ value was 0.041 to 1.65 seconds and for $\tau_{2}$ it was from 263 to 1284 seconds [1], [10]. The values of theses constants are in good agreement with our results, but the wide range of values reported in literature suggests that these constants are sensitive to the experimental protocol (e.g., the strain rates), analytical approach utilized as well as differences between tendons isolated from different species. Such a degree of variability of constants shows that results cannot be compared between tissues and laboratories. Elliott et al. [10] utilized the QLV theory to determine properties of tendon from mouse tail. Samples were immersed in $37^{\circ} \mathrm{C}$ in PBS bath and elongated to $0.005 \%$ grip-to-grip strain followed by $10 \mathrm{~min}$ of relaxation. Incremental 259 steps of $0.05 \mathrm{~mm}$ with $10 \mathrm{~min}$ of relaxation were repeated until failure. The $A$ value were reported between 128 and $281 \mathrm{MPa}$, the $B$ value was from 2.11 to 3.13 depending of the age of mice. These values were significantly different in comparison to our results, mainly because of species type. For human ankle ligaments tested in step strain tests for strains in the range from 2 to $10 \%$, the $A$ values were reported between 0.14 and $7.18 \mathrm{~N}$, the $B$ values were from 15.50 to $49.63, \tau_{1}$ was from 0.465 to $0.832, \tau_{2}$ was from 6.95 to 14.75 and $\tau_{3}$ was from 131.8 to 244.1 [13]. Except for species differences, also the approach to multiparameters modeling was visible in application of seven model parameters instead of five used in our study. Another issue is difference between the experimental protocol employed in the above reported studies and our study. In this study, direct stress relaxation tests, each conducted at a single axial stretch 
were used instead of incremental stress relaxation tests presented in [10] and [13].

Analyzing the results of this study, several limitations must be taken into consideration. The data examined do not identify all possible nonlinearities in tendons, such as the effects of age, sex, anatomical location, healing, biochemical changes, and others. The model used in this research was assumed as a homogenous, isotropic, incompressible, single phase material. Future investigations should incorporate anisotropy and collagen fiber recruitment of tendons. Another important issue is evaluation of tendon mechanics in the microstructural scale with the use of electron microscopy. Such experimental data collected for tendon tissue and fibril scale provided data necessary to develop appropriate mathematical and computational models.

\section{Conclusions}

In summary, this study reports relaxation behavior in the simulated biological environment and with incorporation of preconditioning to the testing protocol. Fitting the QLV model to stress relaxation data, each collected at different axial stretch provided a very good fit in the range of low physiological strain (3\%). For higher strain levels, robust fit was observed in short-term relaxation, as well as in a plateau phase of stress relaxation. These results showed that the QLV model is reasonable only for low strain levels. Selecting the best constitutive equation for characterization of tendon behavior is also dependent on given experimental data set. Careful planning of stress relaxation tests with consideration of multiple stress or strain levels and analysis of results enable the possibility of choosing single integral models, e.g., the QLV. The quasi-linear viscoelasticity model can be a valuable tool in the field of biomechanics to compare the constants describing injured, diseased, or treated tissues versus those of normal tissues giving possibility of quantitative measurement of differences in viscoelastic behavior. However, the QLV formulation cannot describe all viscoelastic behavior.

\section{References}

[1] Abramowitch S.D., Woo S.L.Y., ClinefF T.D., DebSKi R.E., An evaluation of the quasi-linear viscoelastic properties of the healing medial collateral ligament in a goat model, Annals of Biomedical Engineering, 2004, 32, 329-335.
[2] Bonifasi-Lista C., Lakez S.P., SMall M.S., Weiss J.A., Viscoelastic properties of the human medial collateral ligament under longitudinal, transverse and shear loading, Journal of Orthopaedic Research, 2005, 23, 67-76.

[3] CARNiEl T.A., FANCELlo E.A., A transversely isotropic coupled hyperelastic model for the mechanical behavior of tendons, Journal of Biomechanics, 2017, 54, 49-57.

[4] Ciarletta P., Micera S., Accoto D., Dario P., A novel microstructural approach in tendon viscoelastic modelling at the fibrillar level, Journal of Biomechanics, 2006, 39, 2034-2042.

[5] DAVIS F.M., DE ViTA R., A nonlinear constitutive model for stress relaxation in ligaments and tendons, Annals of Biomedical Engineering, 2012, 40, 2541-2550.

[6] DeFrate L.E., Li G., The prediction of stress-relaxation of ligaments and tendons using the quasi-linear viscoelastic model, Biomechanics and Modeling in Mechanobiology, 2007, 6, 245-251.

[7] Duenwald S.E., Vanderby Jr R., LaKes R.S., Constitutive equations for ligament and other soft tissue: evaluation by experiment, Acta Mechanica, 2009, 205, 23-33.

[8] Duenwald S.E., Vanderby Jr R., Lakes R.S., Stress relaxation and recovery in tendon and ligament: Experiment and modeling, Biorheology, 2010, 47, 1-14.

[9] Duenwald S.E., VANDERBy R., LAKes R.S., Viscoelastic relaxation and recovery of tendon, Annals of Biomedical Engineering, 2009, 37, 1131-1140.

[10] Elliott D.M., Robinson P.S., Gimbel J.A., SARVer J.J., AвBoud J.A., Iozzo R.V., SosLowsky L.J., Effect of altered matrix proteins on quasilinear viscoelastic properties in transgenic mouse tail tendons, Annals of Biomedical Engineering, 2003, 31, 599-605.

[11] FANG F., LAKE S.P., Modelling approaches for evaluating multiscale tendon mechanics, Interface Focus, 2016, 6, 20150044.

[12] FunG Y.C., The Meaning of the constitutive equation, [in:] Biomechanics: mechanical properties of living tissues, Springer-Verlag, New York 1993, 23-65.

[13] FunK J., Hall G.W., Crandall J., Pilkey W.D., Linear and quasi-linear viscoelastic characterization of ankle ligaments, Journal of Biomechanical Engineering, 2000, 122, 15-22.

[14] Johnson G.A., Tramaglini D.M., Levine R.E., Ohno K., CHOI N.Y., Woo S.L., Tensile and viscoelastic properties of human patellar tendon, Journal of Orthopaedic Research, 1994 12, 796-803.

[15] Khayyeri H., Gustafsson A., Heuijerjans A., Matikainen M.K., Julkunen P., Eliasson P., AsPenberg P., IsAKSSON H., A fibre-reinforced poroviscoelastic model accurately describes the biomechanical behaviour of the rat Achilles tendon, PLoS ONE, 2015, 10 (6), e0126869.

[16] LiBER-KNeć A., ŁaGAN S., Experimental and Constitutive Approaches for a Study of Mechanical Properties of Animal Tendons, Advances in Intelligent Systems and Computing, 2020, 1033, 288-297.

[17] Maganaris C.N., NARICI M.V., Mechanical properties of tendons, Tendon injuries, Springer, USA, 2005, 14-21.

[18] Oftadeh R., Connizzo B.K., Nia H.T., Ortiz C., GRODZINSKY A.J., Biological connective tissues exhibit viscoelastic and poroelastic behavior at different frequency regimes: Application to tendon and skin biophysics, Acta Biomaterialia, 2018, 70, 249-259.

[19] Provenzano P.P., LAKES R.S., CorR, D.T., VANDERBY Jr R., Application of nonlinear viscoelastic models to describe ligament behavior, Journal of Biomechanical Engineering, $2002,1,45-57$. 
[20] SHEARER T., A new strain energy function for the hyperelastic modelling of ligaments and tendons based on fascicle microstructure, Journal of Biomechanics, 2015, 48, 290-297.

[21] Shepard J.H., Legerlotz K., Demirci T., Klemt C., Riley G.P., SCREEN H.R.C., Functionally distinct tendon fascicles exhibit different creep and stress relaxation behavior, Proceedings of the Institution of Mechanical Engineers, Part H: Journal of Engineering in Medicine, 2014, 228, 49-59.

[22] Sopakayang R., A new viscoelastic model for preconditioning in ligaments and tendons, Proceedings of the World Congress on Engineering, 2013, 3, 1717-1722.
[23] Teramoto A., Luo Z.P., Temporary tendon strengthening by preconditioning, Clinical Biomechanics, 2008, 23, 619-622.

[24] Walden G., Liao X., Donell S., Raxworthy M.J., Riley G.P., SAEED A., A clinical, biological, and biomaterials perspective into tendon injuries and regeneration, Tissue Engineering Part B Reviews, 2017, 23, 44-58.

[25] Wren T.A.L., Yerby S.A., BEAUPRÉ G.S., CARTER D.R., Influence of bone mineral density, age and strain rate on the failure mode of human Achilles tendons, Clinical Biomechanics, 2001, 16, 529-534. 